\title{
Multiple Vehicle Cooperative Localization Under Random Finite Set Framework
}

\author{
Feihu Zhang, Hauke Stähle, Guang Chen, Christian Buckl and Alois Knoll
}

\begin{abstract}
This paper presents a new multiple vehicle cooperative localization approach based on Random Finite Set (RFS) theory. Assuming vehicles are equipped with proprioceptive and exteroceptive sensors to localize the positions, a solution based on RFS statistics is therefore proposed to consider the whole group behavior instead of each vehicle. For this, we rely on Probability Hypothesis Density (PHD) filtering. Compared to other methods, our approach presents a recursive filtering algorithm that provides dynamic estimation of multiple vehicle states. The proposed method addresses the current challenges in multiple vehicle cooperative localization domain such as communication bandwidth issue, data association uncertainty and the over-convergence problem.

A comparative study based on simulations demonstrates the reliability and the feasibility of the proposed approach in large scale environments.
\end{abstract}

\section{INTRODUCTION}

Within the past decade, accurate global localization has become a hot issue in the intelligent vehicle research domain, not only for developing advanced driver assistance systems, but also for achieving autonomous driving.

Single vehicle localization is often performed by fusing both proprioceptive and exteroceptive sensors presented in [1]. GPS [2][3], cameras [4] and laser scanners [5][6] are often used for localization. However, with the developed inter-vehicle communication technology [7], the information exchange among the vehicles becomes available. With a wireless communication device, the multiple vehicle cooperative localization can be improved by taking advantage of information sharing. It is obvious that cooperative localization will present more precise estimation than individual localization. Various methods are investigated for cooperative localization such as Extended Kalman Filter [8][9][10], Bayesian formalism [11], Markov Localization [12], Maximum Likelihood Estimation [13] and Maximum A Posteriori Estimation [14][15]. However, there are still open issues which are introduced in the following:

- The communication bandwidth issue.

The communication bandwidth issue is introduced by Nerurkar [16]. The wireless transceivers used for information exchanging may have low bandwidth due to the restriction of the power. However, a high communication bandwidth for transmitting the estimation state including large covariance matrices, is often required by the standard fusion methods.

Feihu Zhang, Hauke Stähle, Guang Chen and Alois Knoll are with the Technische Universität München, Garching bei München, Germany, e-mail: feihu.zhang@tum.de, \{staehle,guang,knoll $\} @$ in.tum.de.

Christian Buckl is with fortiss $\mathrm{GmbH}$, München, e-mail: buck1@fortiss.org.
Specially, as the number of vehicles increases, the network might get overloaded and thus unusable. Therefore, the optimally solution is that the wireless transceiver only transmits their observations while the system estimates the states with the corresponding covariances.

- The uncertainty issue.

Data association plays an important role in multiple vehicle localization [17]. With the developing of the V2V communication systems, vehicles are able to localize and identify the other members of the group correctly. Various aspects of inter-vehicle communication are surveyed in [18]. However, in a multiple vehicle cooperative localization scenario, measurements are often consist of clutters which is hard to identify. In order to build up a more generic localization framework, addressing the association uncertainty is therefore required. In addition, the localization system should be more dynamic to the constrains. For instance, sensors may often delay, or temporarily unavailable. Therefore, the dynamic structure of the vehicles should be considered-not only the states, but also the number of the vehicles.

- The over-convergence issue.

Over-convergence often happens in the fusion process [19]. It is described as follows: Each vehicle can estimate the pose information of the whole group and broadcast this information to the others. In the case of circular update, for example, a scenario in which the vehicle $i$ detects the vehicle $j$, the vehicle $j$ can update its pose estimation with the observation of the vehicle $i$. However, the observation from vehicle $j$ cannot be used by the vehicle $i$ to update its pose estimation. This is due to the stochastic interdependence between the estimations of the two vehicles. Various methods are investigated to the over-convergence issue [19], [20]. However, the solutions are with strong assumptions which doesn't satisfy the above issues, e.g. bandwidth issue and data association issue.

In this paper, we propose a method for multiple vehicle cooperative localization based on Probability Hypothesis Density (PHD) filter [21]. In the PHD filter, the collection of individual vehicles is treated as set-valued state, and the collection of individual measurements is treated as setvalued observation. Modeling set-valued state and set-valued observation as RFS provides a new view on the multiple vehicle cooperative localization.

The general solution of the proposed approach is as follows: The measurements from both proprioceptive and exteroceptive sensors are projected to a global plane that consist the observation set. The PHD filter recursively estimates the 
dynamic states according to the observations.

The benefits of the proposed approach are threefold:

First, the communication bandwidth is bounded. Since the PHD filter is a recursive Bayes filter which operates on the set space, vehicles only transmit their observations (thus the amount of exchanged data is greatly reduced), which promises the communication ability. As mentioned above, the optimally solution for inter-vehicle communication only transmits observations which minimizes the bandwidth. Compared to other methods, the proposed approach has the lowest consumption requirements for the communication bandwidth.

Second, it eliminates the uncertainty problem. Modeling set-valued state not only allows to dynamically estimate each vehicle's state but also the number of the vehicles. The proposed approach illustrates its flexibility and reliability under extreme conditions such as sensor delay, or temporarily unavailable.

Third, it avoids the over-convergence issue. Measurements are collected as set-valued observation to predict and update the set-valued state under RFS framework, which avoids the process of calculating the correlations between different sources. Furthermore, the proposed approach addresses the above three issues simultaneously while takes up little resources.

The advantages of the proposed method are demonstrated, especially through a performance comparison with an existing cooperative localization method [19].

The remainder of this paper is structured as follows: Sec. II briefly describes the multiple vehicle cooperative localization. Sec. III introduces the proposed approach with the implement details. Sec. IV presents experimental results. Finally, the paper is concluded in Sec.V.

\section{BACKGROUND DESCRIPTION}

The description of the multiple vehicle cooperative localization is as follows:

- Each vehicle is able to localize itself according to an absolute reference. Here we assume that the measurements are formatted by the location and the orientation in a $2 \mathrm{D}$ global coordinate.

- Each vehicle is able to measure the relative position of the other vehicles. Here we assume that the measurements are formatted by the range and bearing in a $2 \mathrm{D}$ local coordinate.

- Vehicles are equipped with communication transceivers for information exchanging. Here we assume that the communication can be affected by the delays or can be temporarily unavailable.

- The communication network does not have the identify ability regarding to the data association issue. Each vehicle observes the whole environment and transmits its observations over the network. There is no prior information regarding to the data association issue provided by the inter-vehicle communication.

The goal of multiple vehicle cooperative localization is to take the whole information into account to acquire the most accurate positions.

Much work has been done for cooperative localization by using a centralized extended Kalman filter [8][10]. The state of the group is viewed as a single system. The localization is obtained by exchanged data from each vehicle. The Kalman filter reduces the uncertainty of the localization by information sharing. However, the disadvantage is the large quantity of transmitted data. This quantity grows exponentially with vehicle increases. In addition, for a dynamic multiple vehicle structure, the Kalman filter requires an association process which takes huge resources.

Instead of a centralized architecture, a decentralized solution is investigated, where multiple fusion centers exist and each of them handles only local information (only the observed neighbors). However, the computational demand is very high. In addition, it often leads to the over-convergence problem when handling inter-estimate correlation among various sources. Since the over-convergence problem is caused by inter-estimation correlation, a natural idea for addressing this problem is investigated-controlling of the data flow within the vehicle network. Howard [11] maintains a heuristic method based on a dependency tree to update the distribution dependencies. However, the author assumes that distributions are only dependent on the distribution that was last used and are independent on the others. This assumption is restrictive as circular updates can still occur. The authors in [19] propose a state exchange based method which only allows independent estimates to be shared within the network. However, communication and computation demand is also increased. The authors in [20] proposed a covariance intersection filter [22] to handle the inter-estimate correlation issue. Similar work can also be found in [23][24] which exploit the advantage of estimation. However, as mentioned above, the computational complexity becomes an issue when vehicles increase.

It is still a challenge to address the whole issues, both centralized and decentralized architectures. In the next section we will present the PHD filter under RFS framework to address the above issues. The proposed approach also takes into account the possible delays which often appears in real scenarios.

\section{THE PROBABILITY HYPOTHESIS DENSITY FILTER}

The PHD filter based on Random Finite Set theory is proposed because of its superior performance in multiple targets tracking domain.

\section{A. Overview on RFS Statistics}

The Random Finite Set (RFS) is a hidden markov chain model with set-valued state and set-valued observation while the PHD filter is a predict and correct framework for recursive Bayesian filtering in such a RFS formulation. A comparison of the RFS approach and traditional multiple-target tracking methods has been given in [25]. In the PHD filter, the collection of individual targets is treated as set-valued state, and the collection of individual observations is treated 


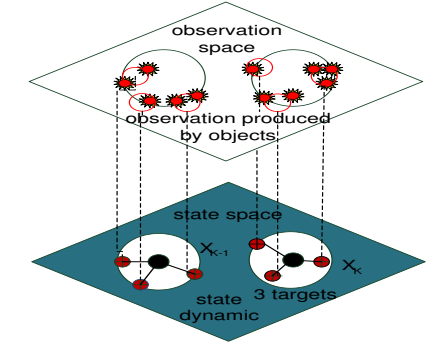

Fig. 1. Set-valued states and set-valued observations

as set-valued observation. Fig. 1 is a basic introduction of the PHD filter which illustrates that the observations and the corresponding states are considered on a single valued space at each step [26]. The PHD filter operates on the set space and avoids the combinatorial problem which arises from data association.

The Gaussian Mixture Probability Hypothesis Density (GM-PHD) filter is a closed form implementation of the PHD filter, which is based on the Bayesian estimation framework utilizing random finite sets as the mathematical backbone [27].

Our previous work was considered the GM-PHD filter as a solution to visual odometry [4], [28] and lane marking extraction [29] in urban environment. The proposed approach estimated the states of the interested features without concerning the association issues on consecutive frames.

B. Kalyan [30] and John. M [31] implemented the PHD filter in the field of the simultaneous localization and mapping (SLAM) problem. Results illustrates that the PHD filter is an effective solution to the SLAM problem. In addition, Moratuwage et. al. implemented the PHD filter in multiple vehicle SLAM problem [32]. However, the above work neither addresses the bandwidth issue nor considers both proprioceptive and exteroceptive sensors' uncertainties. In this paper, the PHD filter is applied for multiple vehicle cooperative localization by utilizing the converted measurement approach [33].

\section{B. Mathematic Background of the PHD Filter}

The RFS is an approximation to alleviate the computational intractability of the optimal multi-target Bayes filter, proposed by Mahler [21].

The targets in a multi-target scenario at time $k$ are represented as a finite set of vectors $\mathbf{x}_{k, 1}, \ldots, \mathbf{x}_{k, N(k)}$ which takes values from the state space $\mathcal{X} \in \mathbb{R}^{n_{\mathrm{x}}}$. Similarly the observations are represented as a finite set of vectors $\mathbf{z}_{k, 1}, \ldots, \mathbf{z}_{k, M(k)}$ which takes values from the observation space $\mathcal{Z} \in \mathbb{R}^{n_{\mathbf{z}}} . N(k)$ and $M(k)$ represent the number of targets and observations at time $k$ respectively. These finite sets are known as the multi-target state and observation:

$$
\begin{aligned}
X_{k} & =\left\{\mathbf{x}_{k, 1}, \ldots, \mathbf{x}_{k, N(k)}\right\} \in \mathcal{F}(\mathcal{X}) \\
Z_{k} & =\left\{\mathbf{z}_{k, 1}, \ldots, \mathbf{z}_{k, M(k)}\right\} \in \mathcal{F}(\mathcal{Z})
\end{aligned}
$$

where $\mathcal{F}(\mathcal{X})$ and $\mathcal{F}(\mathcal{Z})$ denote the sets of all finite subsets of $\mathcal{X}$ and $\mathcal{Z}$, respectively.

The model must encapsulate the time varying numbers of targets in a multi-target scenario. Also the model must consider sensor imperfections such as missed detections and false alarms. The multi-target state is modeled as the union of different random finite sets:

$$
X_{k}=\left[\bigcup_{\zeta \in X_{k-1}} S_{k \mid k-1}(\zeta)\right] \cup \Gamma_{k}
$$

$S_{k \mid k-1}$ represents the targets that have survived from the previous time increment $k-1$. It is modeled as a Bernoulli RFS which means targets can either survive with probability $P_{S, k}\left(\mathbf{x}_{k-1}\right)$ and take on the new value $\left\{\mathbf{x}_{k}\right\}$ with probability density $f_{k \mid k-1}\left(\mathbf{x}_{k} \mid \mathbf{x}_{k-1}\right)$ or die and become the empty set $\varnothing$ with probability $1-P_{S, k}\left(\mathbf{x}_{k-1}\right) . \Gamma_{k}$ represents targets which are spontaneously born at the current time $k$. It is modeled as a Poisson RFS which is specified by a mean birth rate and spatial birth density, or equivalently by its PHD or intensity $\gamma_{k}(\cdot)$ where the mean birth rate is $\int \gamma_{k}(\mathbf{x}) d \mathbf{x}$ and the spatial birth density is $\gamma_{k}(\cdot) / \int \gamma_{k}(\mathbf{x}) d \mathbf{x}$.

Similarly the set observation $Z_{k}$ can be seen as the union of two random finite sets:

$$
Z_{k}=\left[\bigcup_{\mathbf{x} \in X_{k}} \Theta_{k}(\mathbf{x})\right] \cup K_{k}
$$

$\Theta_{k}$ represents the measurements that originate from the targets and is modeled as a Bernoulli RFS which generates a detection with probability $P_{D, k}\left(\mathbf{x}_{k}\right)$ and yields the measurement $\left\{\mathbf{z}_{k}\right\}$ with probability density $g_{k}\left(\mathbf{z}_{k} \mid \mathbf{x}_{k}\right)$ or results in a missed detection yielding an empty measurement set $\varnothing$ with probability $1-P_{D, k}\left(\mathbf{x}_{k}\right)$.

$K_{k}$ represents the set of false alarms or clutter and is modeled as a Poisson RFS, specified by its intensity $\kappa_{k}(\cdot)$ where the mean clutter rate is $\int \kappa_{k}(\mathbf{z}) d \mathbf{z}$ and the spatial clutter density is $\kappa_{k}(\cdot) / \int \kappa_{k}(\mathbf{z}) d \mathbf{z}$.

Using these random finite set models it is possible to construct multi-target dynamical and observation models analogous to the single-target case. Randomness in $X_{k}$ and $Z_{k}$ can be encapsulated into a multi-target transition density and multi-target observation likelihood.

Under the above models, the multi-target Bayes filter propagates the posterior multi-target density $\pi_{k}\left(\cdot \mid Z_{1: k}\right)$ recursively in time. However, due to its combinatorial nature, it is intractable in most applications. To alleviate this, the PHD filter propagates the first moment or PHD $D_{k}(\cdot)$ of multi-target posterior density $\pi_{k}(\cdot)$.

The PHD recursion is given by :

$$
\begin{gathered}
D_{k \mid k-1}\left(\mathbf{x}_{k}\right)= \\
\int P_{S, k}\left(\mathbf{x}_{k-1}\right) f_{k \mid k-1}\left(\mathbf{x}_{k} \mid \mathbf{x}_{k-1}\right) D_{k-1}\left(\mathbf{x}_{k-1}\right) d \mathbf{x}_{k-1} \\
+\gamma_{k}\left(\mathbf{x}_{k}\right)
\end{gathered}
$$

$$
\begin{aligned}
& D_{k}\left(\mathbf{x}_{k}\right)=\left(1-P_{D, k}\left(\mathbf{x}_{k}\right)\right) D_{k \mid k-1}\left(\mathbf{x}_{k}\right) \\
& +\sum_{z \in Z_{k}} \frac{P_{D, k}\left(\mathbf{x}_{k}\right) g_{k}\left(z \mid \mathbf{x}_{k}\right) D_{k \mid k-1}\left(\mathbf{x}_{k}\right)}{\kappa_{k}(z)+\int P_{D, k} g_{k}(z \mid \zeta) D_{k \mid k-1}(\zeta) d \zeta}
\end{aligned}
$$


Equation (7) illustrates that the integral of the PHD over a certain domain $\Psi$ yields the estimated number of targets $N(k)$ in that domain at time $k$. The PHD is not a probability density and does not necessarily sum to 1 [21].

$$
N(k)=\int_{\Psi} D_{k}\left(\mathbf{x}_{k}\right) d \mathbf{x}_{k}
$$

It is to be noted that the PHD recursion has multiple integrals that have no closed form solutions in general. One of the common approaches to mitigate this problem is to use GM-PHD approximations. The GM-PHD filter [27] is a specialized version of the PHD filter. It assumes that the target's motion and observation process can be modeled as:

$$
\begin{aligned}
f_{k \mid k-1}(\mathbf{x} \mid \zeta) & =\mathcal{N}\left(\mathbf{x} ; F_{k-1} \zeta, Q_{k-1}\right) \\
g_{k}(\mathbf{z} \mid \mathbf{x}) & =\mathcal{N}\left(\mathbf{z} ; H_{k} \mathbf{x}, R_{k}\right)
\end{aligned}
$$

where $\mathbf{x}$ refers to the current state, $\mathbf{z}$ to the current measurement, $\zeta$ to the previous state, $\mathcal{N}(\cdot ; \mathbf{m}, P)$ denotes a Gaussian distribution with mean $\mathbf{m}$ and covariance $P$, $F_{k-1}$ is the state transition matrix, $Q_{k-1}$ is the process noise covariance, $H_{k}$ is the observation matrix, $R_{k}$ is the observation noise covariance. Survival and detection probability are supposed to be constant on the entire observed area:

$$
P_{S, k}(\mathbf{x})=P_{S}, P_{D, k}(\mathbf{x})=P_{D}
$$

Birth targets $\gamma_{k}$ are modeled by a RFS written as a Gaussian mixture:

$$
\gamma_{k}(\mathbf{x})=\sum_{i=1}^{J_{\gamma, k}} \omega_{\gamma, k}^{(i)} \mathcal{N}\left(\mathbf{x} ; \mathbf{m}_{\gamma, k}^{(i)}, P_{\gamma, k}^{(i)}\right)
$$

where $\omega_{\gamma, k}^{(i)}, \mathbf{m}_{\gamma, k}^{(i)}$ and $P_{\gamma, k}^{(i)}$ are the weight, mean and covariance of the birth Gaussians and $J_{\gamma, k}$ is their amount.

If the posterior PHD at time $k-1$ is a Gaussian mixture:

$$
D_{k-1}(\mathbf{x})=\sum_{i=1}^{J_{k-1}} \omega_{k-1}^{(i)} \mathcal{N}\left(\mathbf{x} ; \mathbf{m}_{k-1}^{(i)}, P_{k-1}^{(i)}\right)
$$

then the predicted PHD (5) of time $k$ is a Gaussian mixture

$$
D_{k \mid k-1}(\mathbf{x})=P_{S} \sum_{i=1}^{J_{k-1}} \omega_{k-1}^{(i)} \mathcal{N}\left(\mathbf{x} ; \mathbf{m}_{S, k \mid k-1}^{(i)}, P_{S, k \mid k-1}^{(i)}\right)+\gamma_{k}(\mathbf{x})
$$

where

$\mathbf{m}_{S, k \mid k-1}^{(i)}=F_{k-1} \mathbf{m}_{k-1}^{(i)}, P_{S, k \mid k-1}^{(i)}=Q_{k-1}+F_{k-1} P_{k-1}^{(i)} F_{k-1}^{T}$

and the update PHD equation (6) at time $k$ is also a Gaussian mixture and is given by

$$
D_{k}(\mathbf{x})=\left(1-P_{D}\right) D_{k \mid k-1}(\mathbf{x})+\sum_{z \in Z_{k}} D_{D, k}(\mathbf{x} ; \mathbf{z})
$$

where

$$
D_{D, k}(\mathbf{x} ; \mathbf{z})=\sum_{j=1}^{J_{k \mid k-1}} \omega_{k}^{(j)}(\mathbf{z}) \mathcal{N}\left(\mathbf{x} ; \mathbf{m}_{k \mid k}^{(j)}(\mathbf{z}), P_{k \mid k}^{(j)}\right)
$$

$$
\begin{gathered}
\omega_{k}^{j}(\mathbf{z})=\frac{P_{D} w_{k \mid k-1}^{(j)} q_{k}^{(j)}(\mathbf{z})}{\kappa_{k}(\mathbf{z})+P_{D} \sum_{l=1}^{J_{k \mid k-1}} w_{k \mid k-1}^{(l)} q_{k}^{(l)}(\mathbf{z})} \\
q_{k}^{(j)}(\mathbf{z})=\mathcal{N}\left(\mathbf{z} ; H_{k} \mathbf{m}_{k \mid k-1}^{(j)}, H_{k} P_{k \mid k-1}^{(j)} H_{k}^{T}+R_{k}\right) \\
\mathbf{m}_{k}^{(j)}(\mathbf{z})=\mathbf{m}_{k \mid k-1}^{(j)}+K_{k}^{(j)}\left(\mathbf{z}-H_{k} \mathbf{m}_{k \mid k-1}^{(j)}\right) \\
P_{k}^{(j)}=\left[I-K_{k}^{(j)} H_{k}\right] P_{k \mid k-1}^{(j)} \\
K_{k}^{(j)}=P_{k \mid k-1}^{(j)} H_{k}^{T}\left[H_{k} P_{k \mid k-1}^{(j)} H_{k}^{T}+R_{k}\right]^{-1}
\end{gathered}
$$

\section{Implement issues}

Sec. III-B briefly introduced the mathematic background of the PHD filter. However, there are still open issues for multiple vehicle cooperative localization. For instance, the uncertainties of both proprioceptive and exteroceptive sensors are different. However, the PHD filter requires that the observations should have the same uncertainties when constituting the set-valued observation. With the goal of utilizing the PHD filter, we proposed a converted measurements solution as following:

For the process model, the state $\mathbf{x}_{k}=$ $\left[p_{x, k}, p_{y, k}, \dot{p}_{x, k}, \dot{p}_{y, k}\right]^{T}$ of each vehicle consists of position $\left(p_{x, k}, p_{y, k}\right)$ and velocity $\left(\dot{p}_{x, k}, \dot{p}_{y, k}\right)$. Each vehicle has survival probability $P_{S, k}=0.99$, follows the linear Gaussian dynamics (8) with

$$
F_{k}=\left[\begin{array}{ll}
\mathbf{I}_{2} & \mathbf{I}_{2} \\
\mathbf{0}_{2} & \mathbf{I}_{2}
\end{array}\right], Q_{k}=\delta^{2}\left[\begin{array}{cc}
\mathbf{I}_{2} / 4 & \mathbf{I}_{2} / 2 \\
\mathbf{0}_{2} & \mathbf{I}_{2}
\end{array}\right]
$$

where $\mathbf{I}_{n}$ and $\mathbf{O}_{n}$ denotes, respectively, the $n \times n$ identity and zero matrices, $\delta$ is the standard deviation of the process noise.

For the measurement model we consider the measurements are originated from both proprioceptive and exteroceptive sensors projecting to the ground plane. To map the state to the observation space, the observation matrix is $H_{k}=$ $\left[\mathbf{I}_{2}, \mathbf{0}_{2}\right]$ while the measurement vector is $\mathbf{z}=\left[p_{x}, p_{y}\right]^{T}$.

According to the assumptions in Sec. II, measurements are acquired from both proprioceptive and exteroceptive sensors which can be considered as $Z_{k}^{1}, Z_{k}^{2}$. $Z_{k}^{1}$ is from the proprioceptive sensor acquired by GPS while $Z_{k}^{2}$ is acquired by the observations from other vehicles. In this paper, $Z_{k}^{2}$ is calculated by utilizing the whole sensors (For instance, we measure the vehicles' relative positions according to the exteroceptive sensors. Measurements are then acquired by coordinate transformation with the help of the proprioceptive sensors). In addition, $Z_{k}^{1}$ is with standard deviation $\mathbf{R}_{\mathbf{1}}$ while $Z_{k}^{2}$ is with deviation $\mathbf{R}_{2}$ (According to the converted measurement approach [33] by using the combination process, the uncertainty for each single observation is calculated. The mean value is then represented as the deviation $\mathbf{R}_{\mathbf{2}}$ to the PHD filter). Assuming there are $n$ vehicles for the cooperative localization, the size of the observation set $Z_{k}^{1}$ is $n$ while $Z_{k}^{2}$ is $n(n-1)$ at each step.

Regarding to the categories $Z_{k}^{1}$ and $Z_{k}^{2}$, the PHD filter should be implemented sequentially (since different types 
of measurements have different deviations describing the uncertainties, which is impossible to calculate the posterior PHD simultaneously). A method by applying GM-PHD filter sequentially for each set, which is described as follows [34]:

With the assumptions in III-B, at time $k-1$ we have

$$
D_{k-1}(\mathbf{x})=\sum_{i=1}^{J_{k-1}} \omega_{k-1}^{(i)} \mathcal{N}\left(\mathbf{x} ; \mathbf{m}_{k-1}^{(i)}, P_{k-1}^{(i)}\right)
$$

First, we used assumptions on process equation (8), measurement equation (9) and $D_{k-1}(\mathbf{x})$ to predict the intensity $D_{k \mid k-1}^{1}(\mathbf{x})$ by using the equation (13). Second, we update $D_{k \mid k-1}^{1}(\mathbf{x})$ with set $Z_{k}^{1}$ by equation (14) to obtain the PHD $D_{k}^{1}(\mathbf{x})$ at time $k$ for set $Z_{k}^{1}$. Since $D_{k-1}(\mathbf{x})$ is a Gaussian mixture, $D_{k}^{1}(\mathbf{x})$ is also a Gaussian mixture. Third, for set $Z_{k}^{2}$, we use $D_{k}^{1}(\mathbf{x})$ as the predicted PHD and in the similar way to equation (14), we have

$$
D_{k}(\mathbf{x})=\left(1-P_{D}\right) D_{k \mid k-1}^{1}(\mathbf{x})+\sum_{z \in Z_{k}^{2}} D_{D, k}(\mathbf{x} ; \mathbf{z})
$$

Now, we investigate the number of Gaussian components. For $Z_{k}^{1}$, the number of Gaussian components is

$$
J_{k}^{1}=\left(J_{k-1}+J_{\gamma, k}\right)\left(1+\left|Z_{k}^{1}\right|\right)
$$

while for $Z_{k}^{2}$, the number of Gaussian components is

$$
J_{k}^{2}=J_{k}^{1}\left(1+\left|Z_{k}^{2}\right|\right)
$$

The size of Gaussian components grows explosively which leads to huge resources unavailable. In order to avoid the high computation, Gaussian components that are close together will be merged into one Gaussian at each step [4].

Based on the above procedure, we address the uncertainties issue for the GM-PHD filter in multiple vehicle cooperative localization scenarios. The corresponding positions are therefore estimated during the whole process.

\section{EXPERIMENT}

The simulation was implemented with a number of four vehicles on the ground plane. In simulation, vehicles are equipped with both proprioceptive and exteroceptive sensors observing the whole environment. The inter-vehicle communication is also available to exchange the information on the network. In order to evaluate the performance in real environment, the communication can be affected with delays, or can be temporarily unavailable. Furthermore, vehicles are not able to identify the others according to the V2V communication system. A comparative method in [19] (SECL approach) was also included to evaluate the performances of both approaches.

Figure 2(a) represents the true trajectories of the vehicles, while Fig. 2(b) plots the corresponding measurements. As we can see from Fig. 2(b), four measurements are used to represent each vehicle's position (if sensor delay not happens). One is from $Z_{k}^{1}$ while others are from $Z_{k}^{2}$. There is no association process between the vehicle and the measurements in $Z_{k}^{2}$. In addition, measurements are often considered

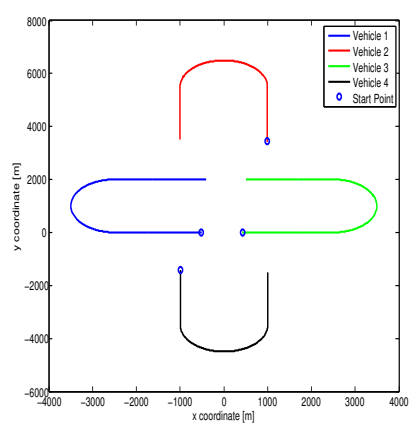

(a)

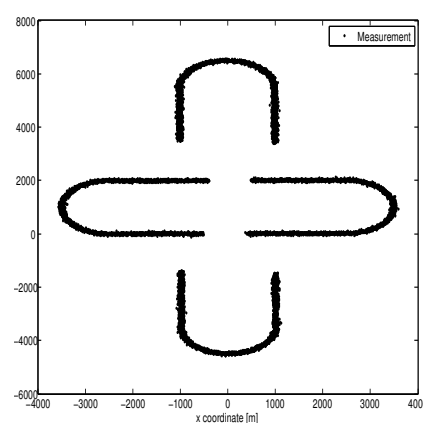

(b)
Fig. 2. The true trajectories and the corresponding measurements

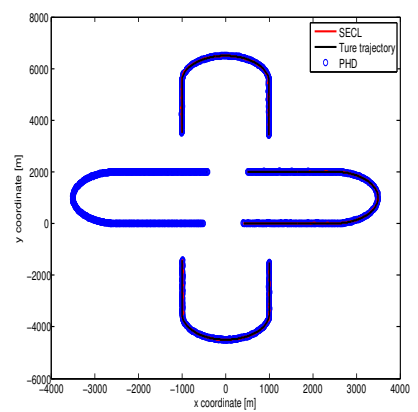

(a)

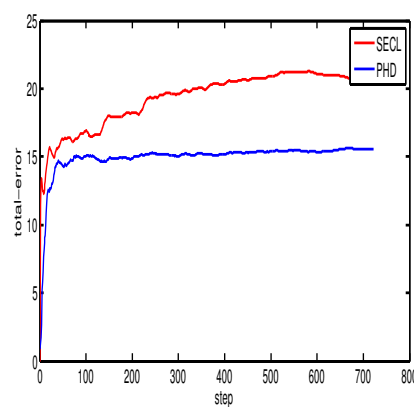

(b)
Fig. 3. The estimated results

as clutter due to the uncertainties. The task of the cooperative localization is to estimate the positions based on those noisy measurements.

Figure 3(a) illustrates the estimations compared with the SECL approach. The estimated PHD states are represented as circles at each step. Compared with standard target tracking methods, the PHD operates on the set space and avoids the association issue. Both, observations and estimations, are setvalued (for example, although the PHD filter can estimate the number of the vehicles and the corresponding states, it does not distinguish them. Thus, the identification of a single vehicle over the whole process is not available). For multiple vehicle cooperative localization, keeping the whole trajectory of each vehicle is out of the scope of this paper.

Figure 3(b) analyzes the performances of both methods by calculating the RMSE (root mean square deviation) value. As mentioned above, the PHD filter operates on the set space which leads to the situation that a comparative result on each vehicle is unavailable. However, in order to compare the performance of the proposed approach, a data association process is implemented to label the PHD estimations with the corresponding vehicles. Furthermore, an evaluation reference is proposed by calculating the RMSE value. The total error is acquired by summing up the RMSE of each vehicle during the whole process:

$$
\text { Error }=\left(x_{\text {est }}-x_{\text {real }}\right)^{2}+\left(y_{\text {est }}-y_{\text {real }}\right)^{2}
$$




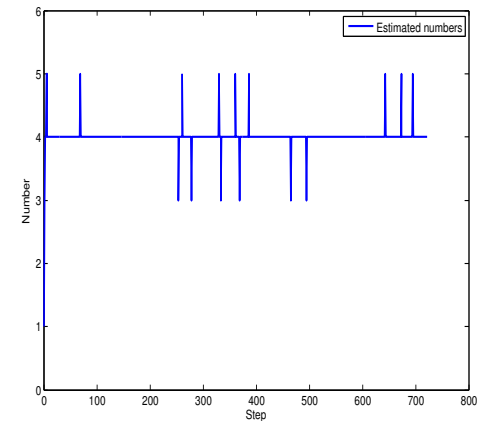

Fig. 4. The estimated numbers of the vehicles

$$
\text { total_error }=\sqrt{\frac{\sum_{j=1}^{n} \sum_{i=1}^{4} \text { Error }_{i}^{j}}{n}}
$$

where $i$ is the index of the vehicles, $n$ is the time index. From Fig. 3 we can see that either PHD or SECL has a certain estimation error compared with the true trajectories. This error is caused by the uncertainties. However, Fig. 3 still illustrates that the overall performance of the PHD filter is better than the SECL approach.

Figure 4 illustrates the number of vehicles that were estimated by the PHD filter. Considering the constrains (such as delay, data association uncertainty, noise, communication unavailability), the number of vehicles is unknown estimating by the PHD filter. The proposed approach overcomes these challenges since the PHD filter operates on the set space which dynamically estimates the states. However, much related works skipped this issue by assuming that prior information was available (e.g. the association between measurements and vehicles, the structure of the group is fixed and the communication network worked error free). Fig. 4 exhibits robustness of the PHD filter for the dynamic structure of the group.

Regarding to the bandwidth and computation issue, the PHD filter still keeps its superiority. As mentioned above, the PHD filter is a predict and correct framework for recursive Bayes filtering which doesn't rely on each estimation and the corresponding covariance for the fusion process. As a matter of fact, vehicles only transmit the measurements to the PHD filter as a set-valued observation to update the set-valued state. The communication bandwidth is therefore reduced.

Assuming each measurement $\left(p_{x}, p_{y}\right)$ takes two communication bits when transmission happens, the PHD filter requires at least $2 n^{2}$ bits bandwidth at each step (There is a total of $n^{2}$ measurements acquired by vehicles which has already mentioned in Sec. III-C, $n$ is the size of the group). However, for the other approaches, e.g. SECL, a fusion process is required which takes huge communication bandwidth. The fusion process utilizes not only the states but also the corresponding covariances to calculate the results. Similar to Sec. III-C, each state is a $4 \times 1$ vector which takes 4 bits including positions and velocities, while the corresponding covariance is a $4 \times 4$ matrix which takes 16 bits respectively. As a conclusion, it requires additional $20 n^{2}$ bits bandwidth for the fusion process while the PHD avoids it (each vehicle is a fusion center which contains the whole states and the corresponding covariances that occupying $20 n$ bits. The requirement of the bandwidth is therefore $20 n^{2}$ bits for the whole vehicles).

In addition, as a recursive Bayes filtering technology, computation complexity is also reasonable for multiple vehicles. A general analytical form to evaluated the calculation complexity of the PHD filter is performed in [35], which illustrates the efficiency of the proposed PHD solution in real time environment.

Compared with decentralized approaches, the overconvergence issue is also avoided since the PHD filter operates on the set space instead of the single state space. Therefore there is no such circular update during the whole process.

The benefits of the PHD filter can be concluded as follows:

First, it reduces and bounds the requirements of the communication bandwidth in a multiple vehicle environment. The inter-vehicle communication system transmits the measurements to the PHD filter which takes little bandwidth. Compared to other methods, the proposed approach has the lowest consumption requirements for the communication bandwidth since each vehicle only transmits its observations.

Second, it works under extreme conditions which often happen in real environments (where the association uncertainty exists, the number of the vehicles is unknown, sensor delays, communication unavailability occurs and the measurement is distracted by noise). The PHD filter not only illustrates the high performance of the localization, but also exhibits the robustness under the dynamic structure of the group.

Third, the over-convergence is addressed. Regarding to the PHD filter, the set-valued state is updated by the setvalued observation recursively. No fusion process is therefore required in the PHD filter, which not only bounds the communication bandwidth and the computation complexity, but also eliminates the influences of the over-convergence.

Because of the superiority mentioned above, we have strong reason to believe that the PHD filter has a great potential in the field of multiple vehicle cooperative localization.

\section{CONCLUSION}

It is very hard to localize a group of vehicles simultaneously. The low communication bandwidth, computational burdens, and data association uncertainty cause the localization complex and unfeasible. In this paper, a PHD filter solution is proposed to the multiple vehicle cooperative localization problem. In comparison to the related work, the whole group of vehicles is viewed as a single set-valued state which contains the poses of the detected vehicles. The measurements are collected as a single set-valued observation which is used to update the behavior of the set-valued state.

The proposed method has been evaluated in simulation and a comparative study has also been carried out. Experimental results exhibit the advantages of the PHD filter for multiple vehicle localization. 
Future work focuses on the evaluation of the proposed approach in a non-synthetic environment.

\section{REFERENCES}

[1] J. Laneurit, R. Chapuis, and F. Chausse, "Accurate vehicle positioning on a numerical map," International Journal of Control, Automation, and Systems, vol. 3, no. 1, pp. 15-31, 2005.

[2] W.-W. Kao, "Integration of gps and dead-reckoning navigation systems," in Vehicle Navigation and Information Systems Conference, 1991, vol. 2, oct. 1991, pp. 635 - 643.

[3] S. Rezaei and R. Sengupta, "Kalman filter-based integration of dgps and vehicle sensors for localization," Control Systems Technology, IEEE Transactions on, vol. 15, no. 6, pp. 1080 -1088, nov. 2007.

[4] F. Zhang, G. Chen, H. Stahle, C. Buckl, and A. Knoll, "Visual odometry based on random finite set statistics in urban environment," in Intelligent Vehicles Symposium (IV), 2012 IEEE, june 2012, pp. 69 -74 .

[5] H. J. Sohn and B. K. Kim, "A robust localization algorithm for mobile robots with laser range finders," in Robotics and Automation, 2005. ICRA 2005. Proceedings of the 2005 IEEE International Conference on, april 2005, pp. 3545 - 3550.

[6] M. Liu, S. Huang, and G. Dissanayake, "Feature based slam using laser sensor data with maximized information usage," in Robotics and Automation (ICRA), 2011 IEEE International Conference on, may 2011, pp. $1811-1816$.

[7] Y. Khaled, M. Tsukada, J. Santa, J. Choi, and T. Ernst, "A usage oriented analysis of vehiclular networks: from technologies to applications," Journal of Communications, pp. 357-368, 2009.

[8] S. Roumeliotis and I. Rekleitis, "Analysis of multirobot localization uncertainty propagation," in Intelligent Robots and Systems, 2003. (IROS 2003). Proceedings. 2003 IEEE/RSJ International Conference on, vol. 2, oct. 2003 , pp. 1763 - 1770 vol.2.

[9] S. Roumeliotis and G. Bekey, "Distributed multirobot localization," Robotics and Automation, IEEE Transactions on, vol. 18, no. 5, pp. $781-795$, oct 2002.

[10] N. Karam, F. Chausse, R. Aufrere, and R. Chapuis, "Cooperative multi-vehicle localization," in Intelligent Vehicles Symposium, 2006 IEEE, 0-0 2006, pp. $564-570$.

[11] A. Howard, M. Mataric, and G. Sukhatme, "Putting the 'i' in 'team': an ego-centric approach to cooperative localization," in Robotics and Automation, 2003. Proceedings. ICRA '03. IEEE International Conference on, vol. 1, sept. 2003, pp. $868-874$ vol.1.

[12] D. Fox, W. Burgard, and S. Thrun, "Markov localization for mobile robots in dynamics environments," Journal of Artificial Intelligence Research, vol. 11, pp. 391-427, 1999.

[13] A. Howard, M. Matark, and G. Sukhatme, "Localization for mobile robot teams using maximum likelihood estimation," in Intelligent Robots and Systems, 2002. IEEE/RSJ International Conference on, vol. 1, 2002, pp. $434-439$ vol.1.

[14] E. Nerurkar, S. Roumeliotis, and A. Martinelli, "Distributed maximum a posteriori estimation for multi-robot cooperative localization," in Robotics and Automation, 2009. ICRA '09. IEEE International Conference on, may 2009, pp. $1402-1409$.

[15] N. Trawny, S. Roumeliotis, and G. Giannakis, "Cooperative multirobot localization under communication constraints," in Robotics and Automation, 2009. ICRA '09. IEEE International Conference on, may 2009, pp. $4394-4400$.

[16] E. D. Nerurkar, K. X. Zhou, and S. I. Roumeliotis, "A hybrid estimation framework for cooperative localization under communication constraints," in Intelligent Robots and Systems (IROS), 2011 IEEE/RSJ International Conference on, sept. 2011, pp. $502-509$.

[17] M. Moratuwage, W. Wijesoma, B. Kalyan, N. Patrikalakis, and P. Moghadam, "Collaborative multi-vehicle localization and mapping in high clutter environments," in Control Automation Robotics Vision (ICARCV), 2010 11th International Conference on, dec. 2010, pp. $1422-1427$.

[18] J. Luo and J.-P. Hubaux, "A survey of inter-vehicle communication," School of Computer and Communication Sciences, EPFL, Switzerland, Tech. Rep., 2004.

[19] N. Karam, F. Chausse, R. Aufrere, and R. Chapuis, "Localization of a group of communicating vehicles by state exchange," in Intelligent Robots and Systems, 2006 IEEE/RSJ International Conference on, oct. 2006, pp. $519-524$.
[20] H. Li and F. Nashashibi, "Cooperative multi-vehicle localization using split covariance intersection filter," in Intelligent Vehicles Symposium (IV), 2012 IEEE, june 2012, pp. 211 -216.

[21] R. Mahler, "Multitarget bayes filtering via first-order multitarget moments," IEEE Transactions on Aerospace and Electronic Systems, no. 4, pp. 1152 - 1178, Vol. 39, Oct. 2003.

[22] S. Julier and J. Uhlmann, "A non-divergent estimation algorithm in the presence of unknown correlations," in American Control Conference, 1997. Proceedings of the 1997, vol. 4, jun 1997, pp. $2369-2373$ vol.4.

[23] P. Arambel, C. Rago, and R. Mehra, "Covariance intersection algorithm for distributed spacecraft state estimation," in American Control Conference, 2001. Proceedings of the 2001, vol. 6, 2001, pp. 4398 -4403 vol.6.

[24] S. Julier and J. Uhlmann, "Using multiple slam algorithms," in Intelligent Robots and Systems, 2003. (IROS 2003). Proceedings. 2003 IEEE/RSJ International Conference on, vol. 1, oct. 2003, pp. 200 205 vol.1.

[25] I. Goodman, R. Mahler, and H. Nguyen, Mathematics of Data Fusion. Norwell, MA: Kluwer Academic, 1997.

[26] B.-N. Vo, "Random finite sets in stochastic filtering," EEE Department University of Melbourne Australia, Tech. Rep., 2009. [Online]. Available: http://www.ee.unimelb.edu.au/staff/bv/

[27] B.-N. Vo and W.-K. Ma, "The gaussian mixture probability hypothesis density filter,' IEEE Transactions on Signal Processing, no. 11, pp. 4091 -4104, Vol. 54, Nov. 2006.

[28] F. Zhang, H. Stahle, A. Gaschler, C. Buckl, and A. Knoll, "Single camera visual odometry based on random finite set statistics," in Intelligent Robots and Systems (IROS), 2012 IEEE/RSJ International Conference on, Oct., pp. 559-566.

[29] F. Zhang, H. Stahle, C. Chen, C. Buckl, and A. Knoll, "A lane marking extraction approach based on random finite set statistics," in Intelligent Vehicles Symposium (IV), 2013 IEEE, june 2013.

[30] B. Kalyan, W. S. Wijesoma, and K. W. Lee, "Fisst-slam: Finite set statistical approach to simultaneous localization and mapping," International Journal of Robotics Research, no. 2, Sept. 2010.

[31] J. Mullane, B.-N. Vo, M. Adams, and B.-T. Vo, "A random-finite-set approach to bayesian slam," IEEE Transactions on Robotics, no. 2, pp. 268 -282, Vol 27, April 2011.

[32] D. Moratuwage, B.-N. Vo, and D. Wang, "A hierarchical approach to the multi-vehicle slam problem," in Information Fusion (FUSION), 2012 15th International Conference on, july 2012, pp. $1119-1125$.

[33] S. Bordonaro, P. Willett, and Y. Bar-Shalom, "Unbiased tracking with converted measurements," in Radar Conference (RADAR), 2012 IEEE, may 2012, pp. $0741-0745$.

[34] N. T. Pham, W. Huang, and S. Ong, "Multiple sensor multiple object tracking with gmphd filter," in Information Fusion, 2007 10th International Conference on, july 2007, pp. $1-7$.

[35] Z. Wang, X. Xiaobin, L. Weifeng, and W. Chenglin, "Performance analysis and comparison of two classic algorithms in multi-target tracking," Journal of Electronics \& Information Technology, vol. 32, no. 7 , July 2010. 